\title{
Organic Electrochemical Transistors
}

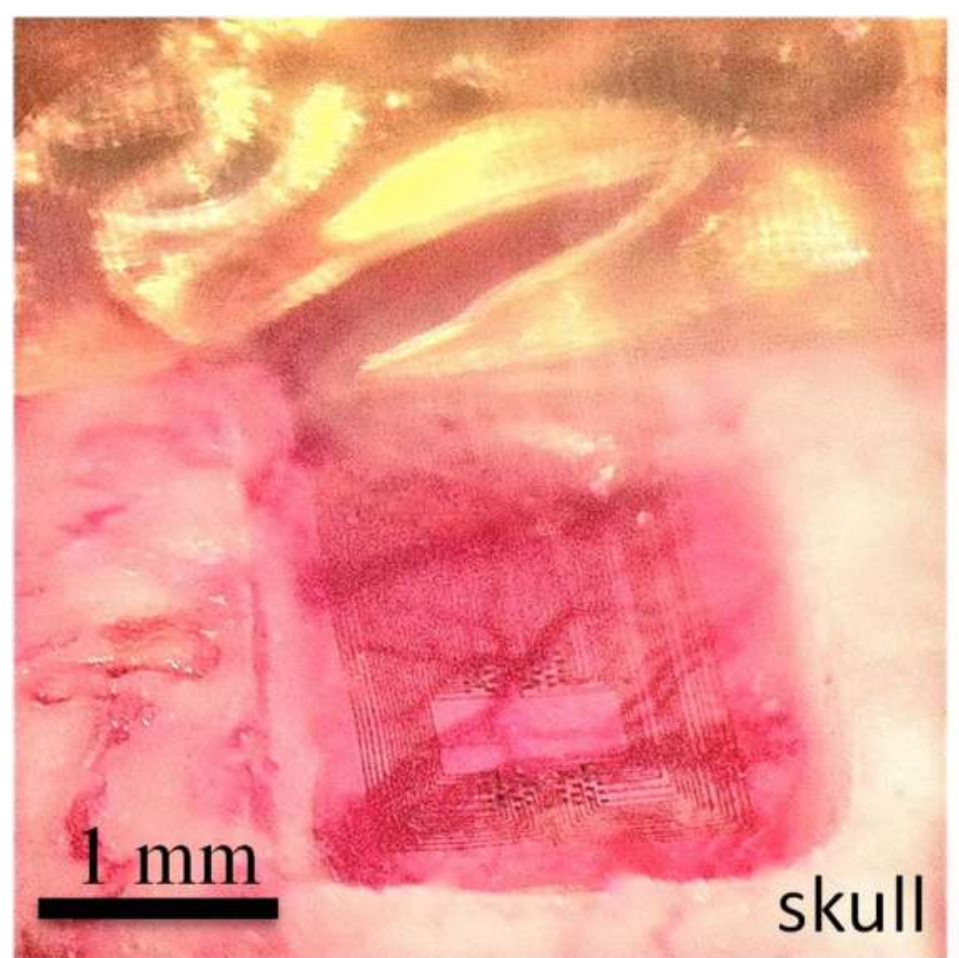

\section{George Malliaras}

Electrical Engineering Division, University of Cambridge 


\section{Outline}

- The organic electrochemical transistor

- Applications in neural recordings

- How do they work?

Conclusions 


\section{Bioelectronics: Coupling biology and electronics}

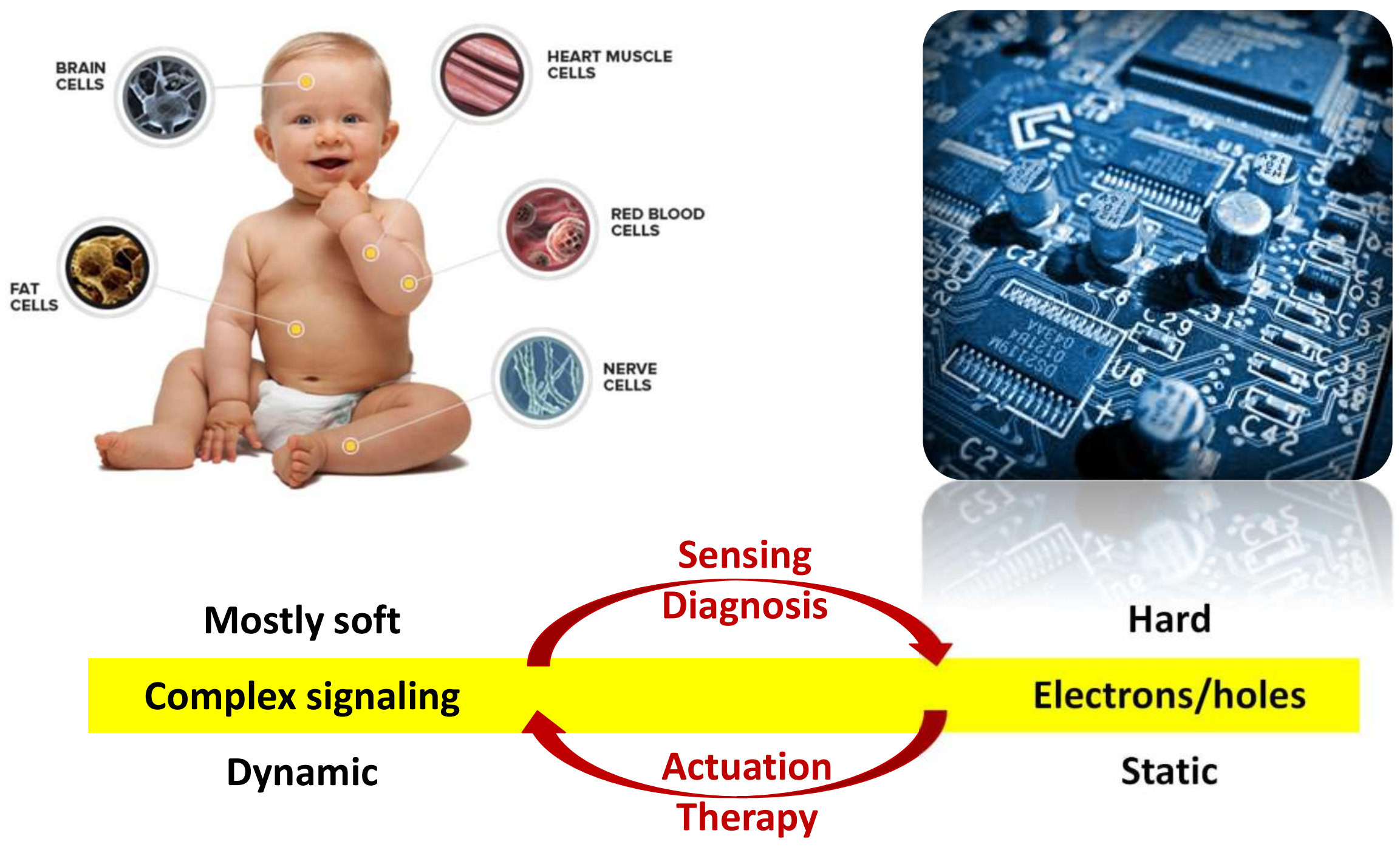




\section{Importance of neural interfacing}

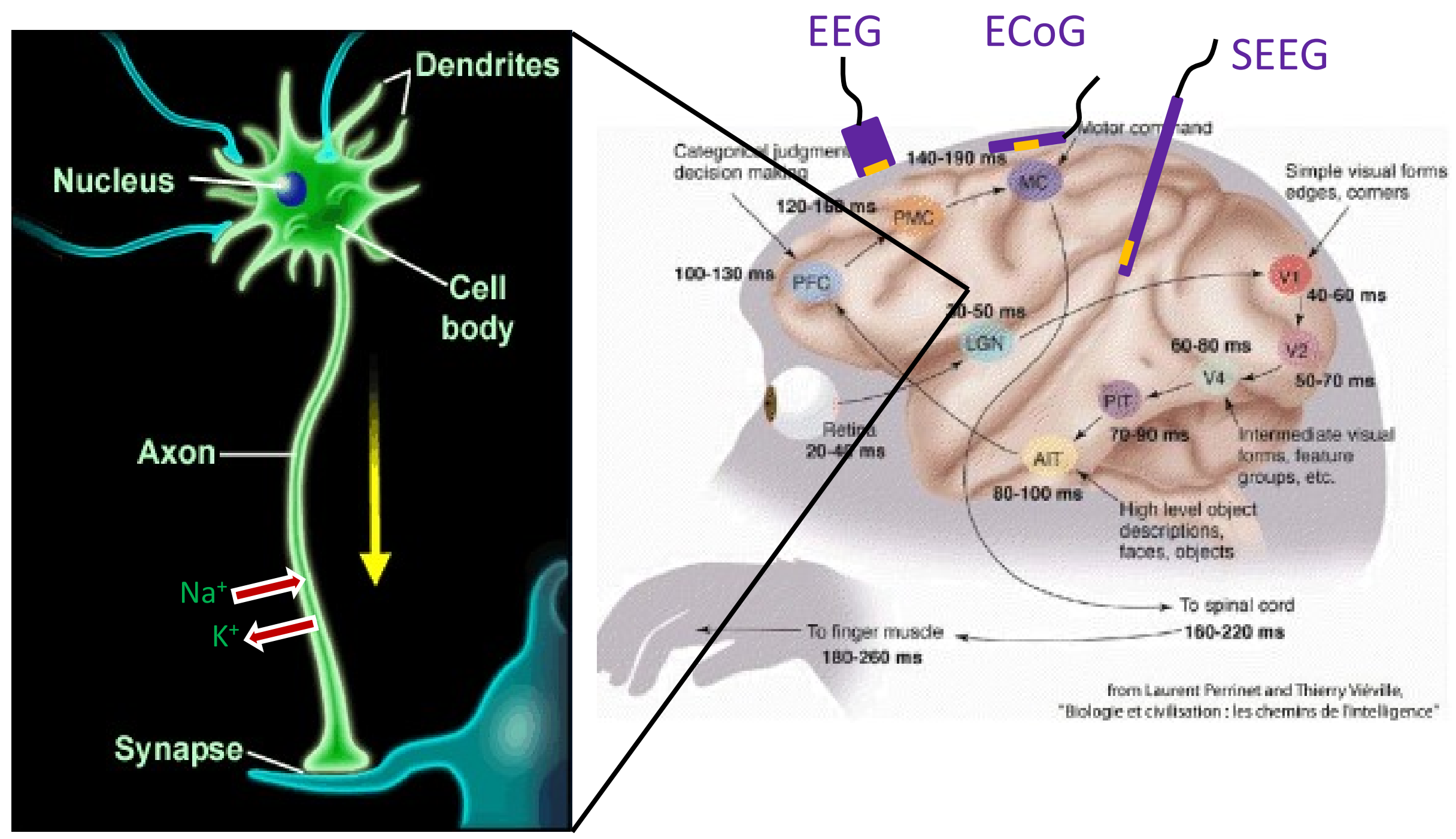




\section{Electrical recording for epilepsy monitoring}

Affects 1-2\% of world population

- Temporal lobe epilepsy (TLE) is most frequent form in adults

- TLE is often drug resistant
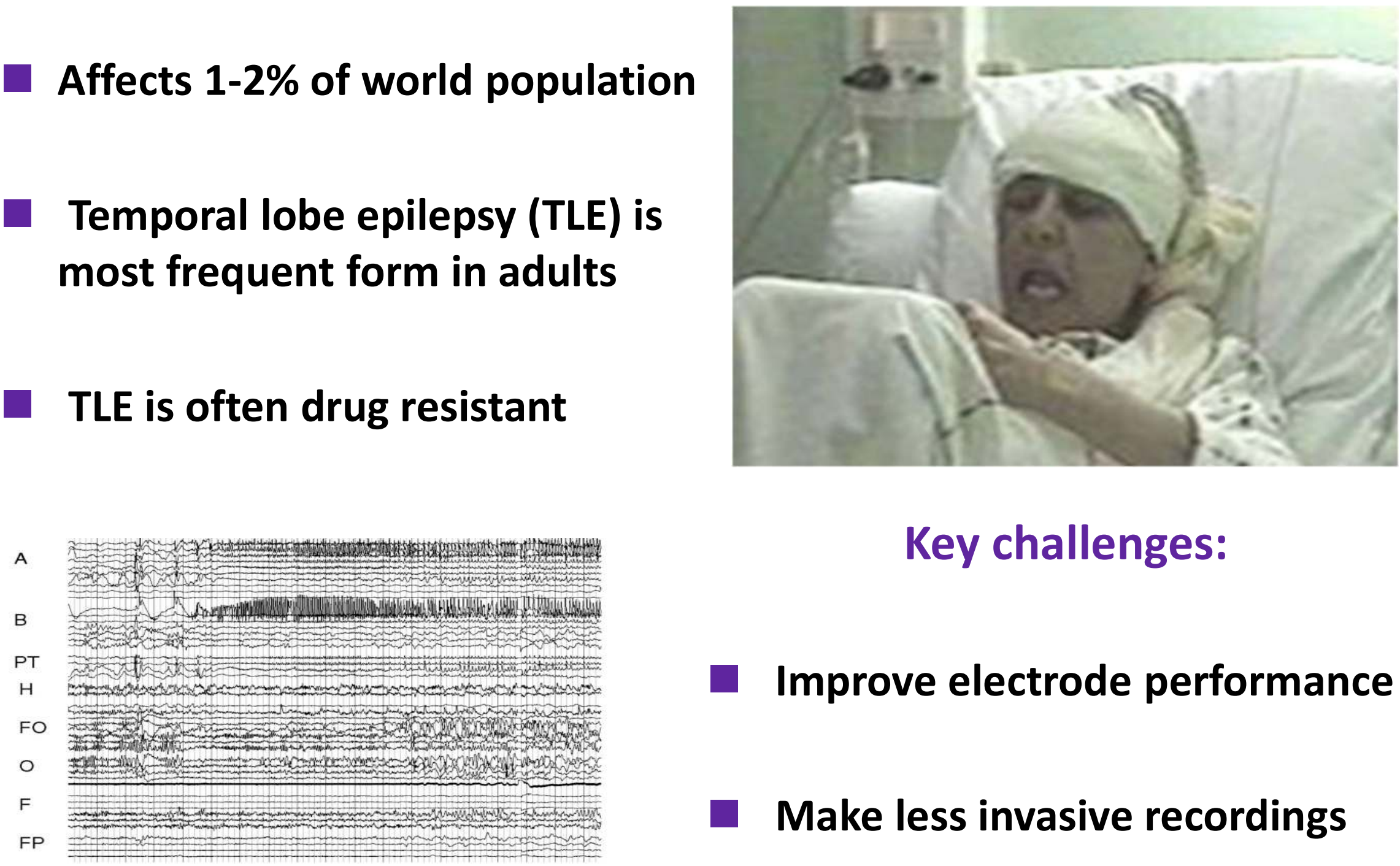

Key challenges:

- Improve electrode performance

Make less invasive recordings 


\section{Brain-Computer Interfaces}

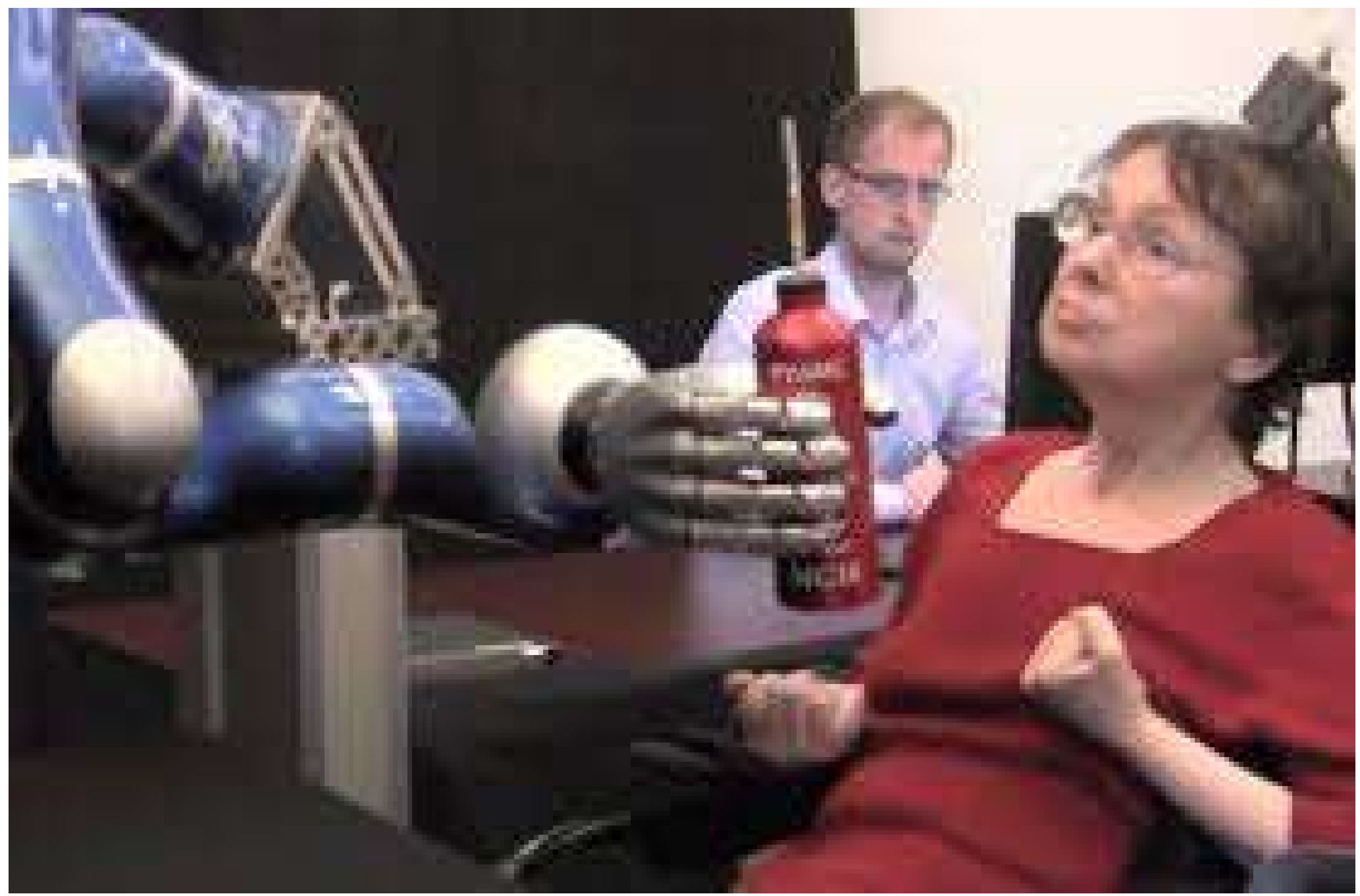




\section{Transistor vs. electrode}
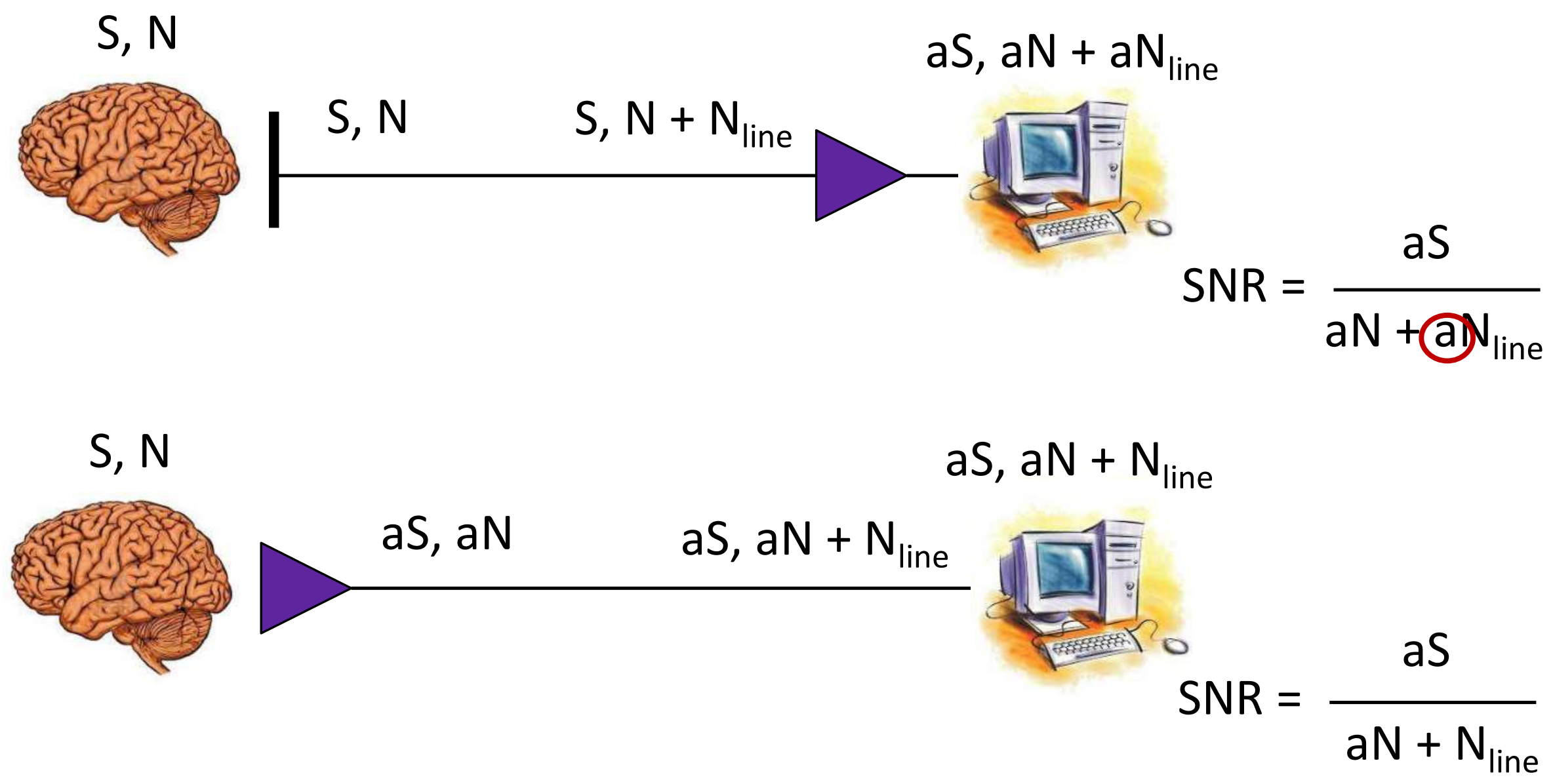

Transistor recordings offer higher SNR 


\section{Recording neural activity with transistors}
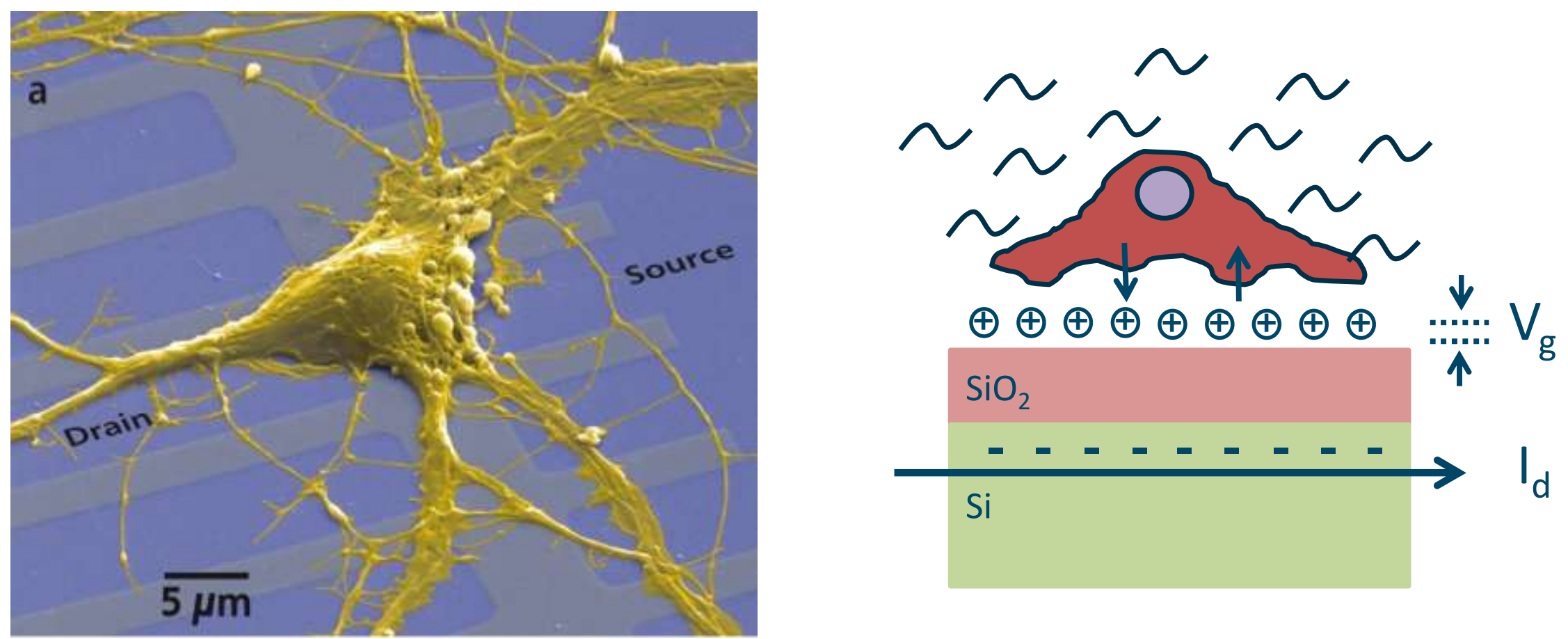

Fromherz group, MPI 


\section{The organic electrochemical transistor (OECT)}

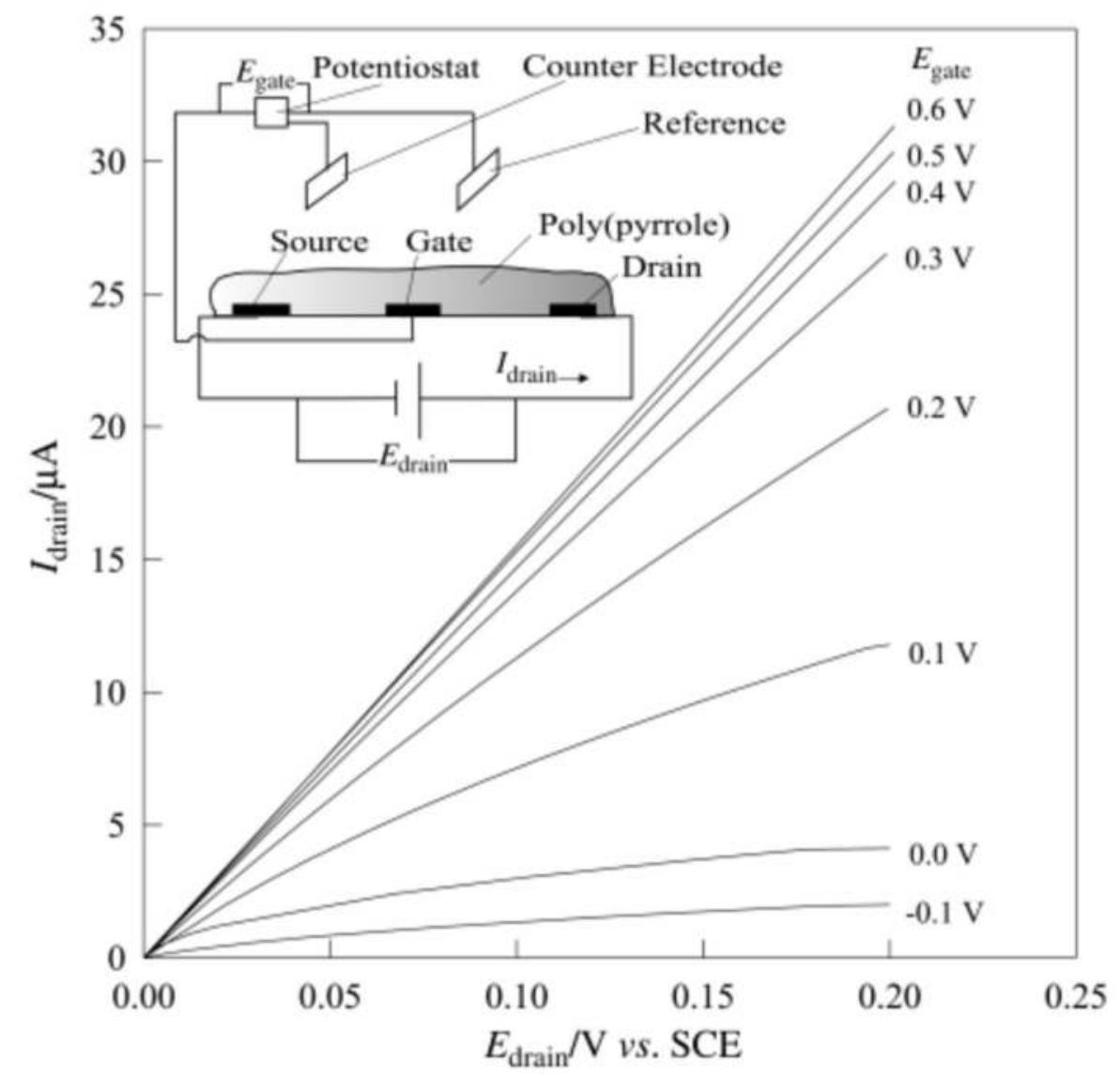

No insulator between channel and electrolyte 


\section{Why organics?}

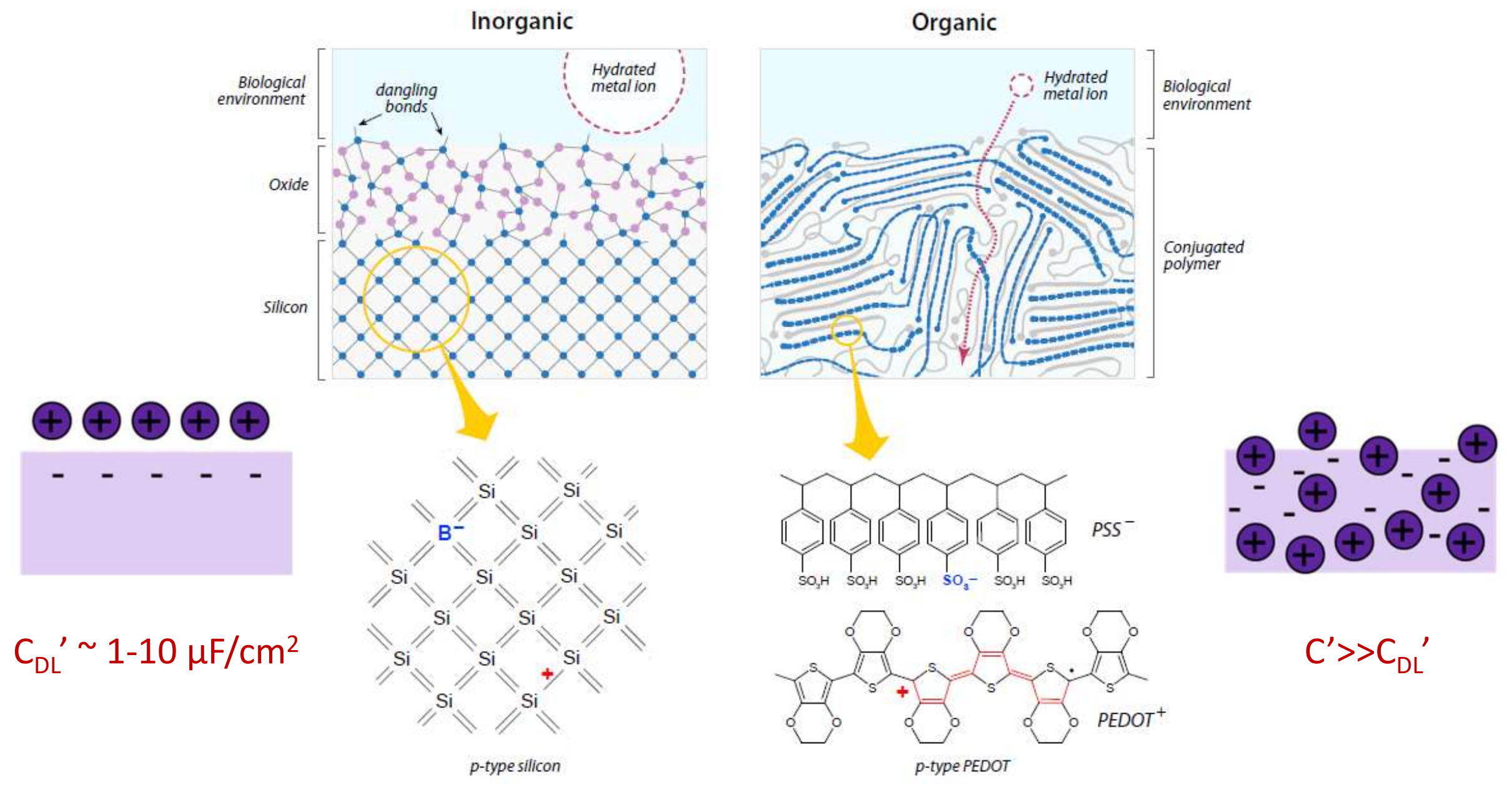

Mixed conductivity leads to novel/state-of-the-art devices 


\section{Molecular engineering of ion injection}
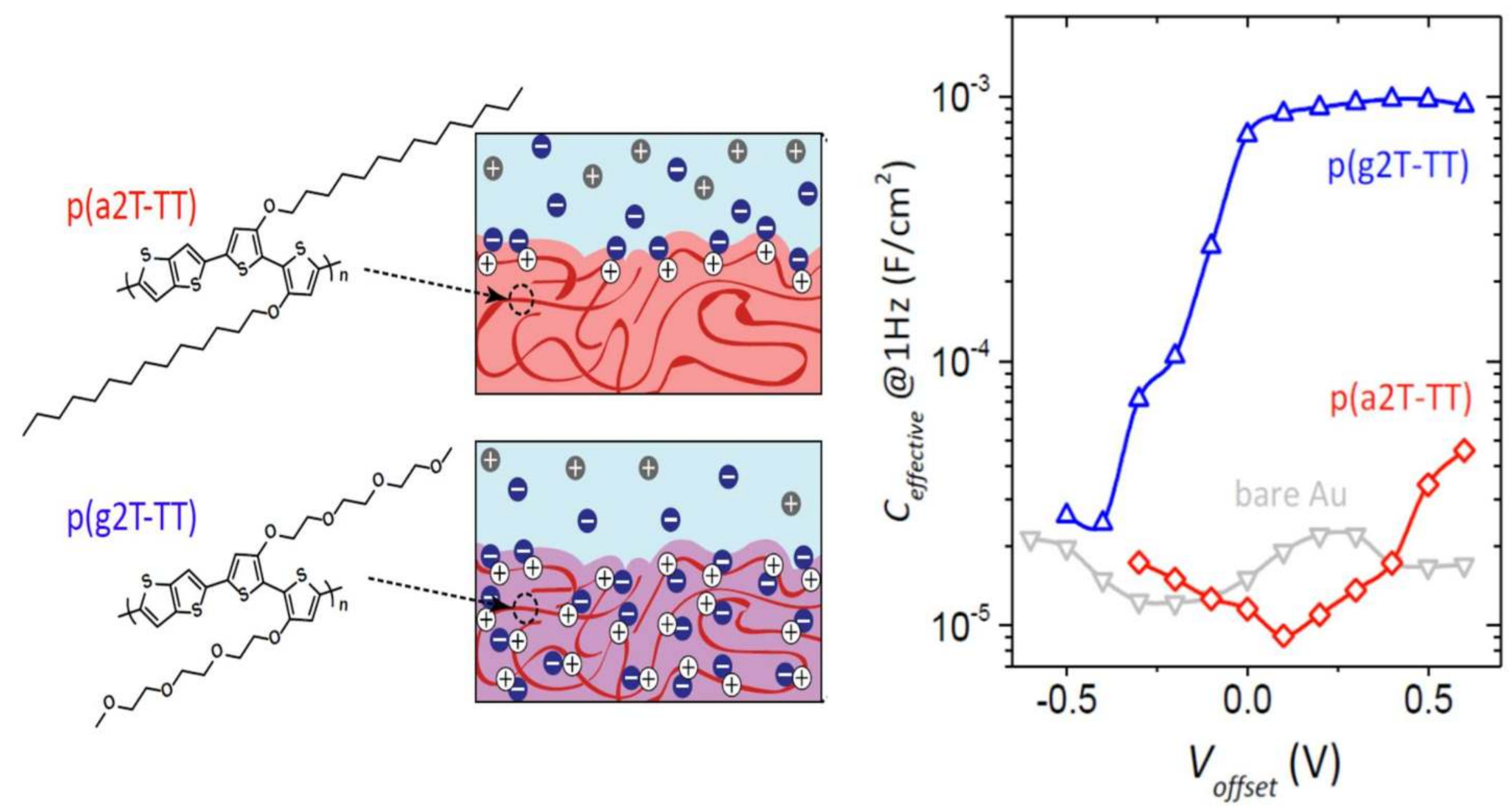

A. Giovannitti, D.R. Sbircea, S. Inal, C.B. Nielsen,

W/ Iain McCulloch (Imperial/KAUST), Jonathan Rivnay (Northwestern) $\begin{gathered}\text { E. Bandiello, D.A. Hanifi, M. Sessolo, G.G. Malliaras, } \\ \text { I. McCulloch, J. Rivnay, PNAS 113, 12017 (2016). }\end{gathered}$ 


\section{Volumetric ion transport in PEDOT:PSS}

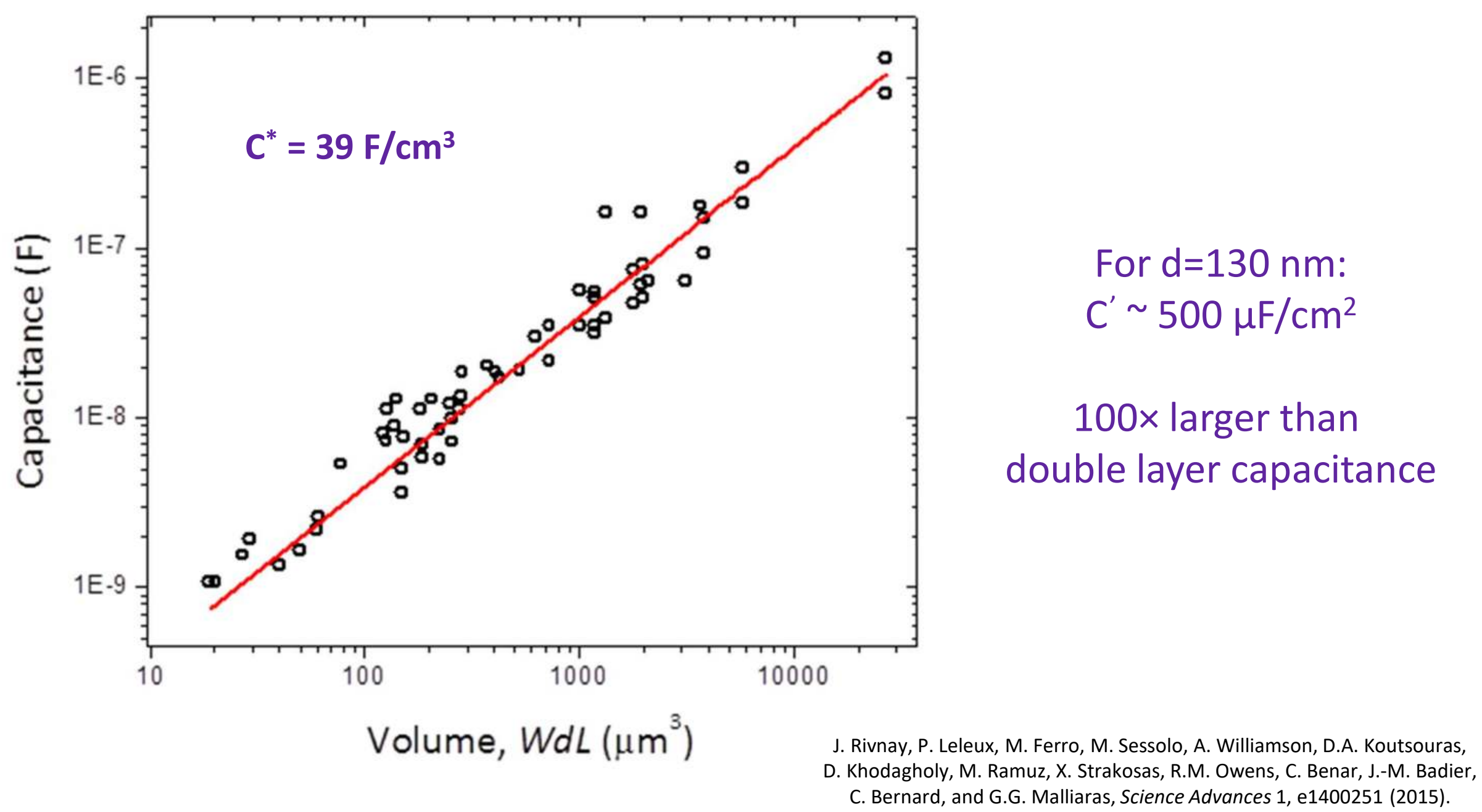




\section{Lithographic fabrication using parylene-C}
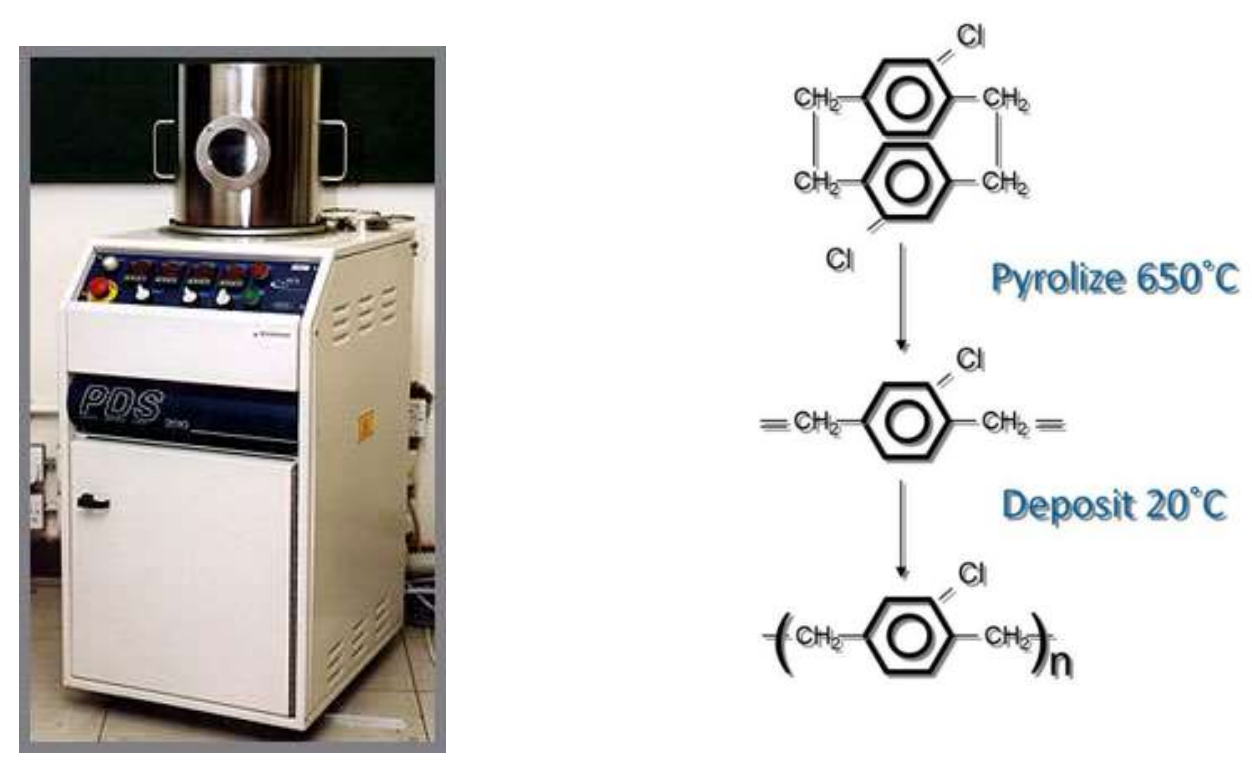

- CVD polymer

- Conformal

- Chemically inert

Parylene deposition

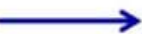

Film deposition
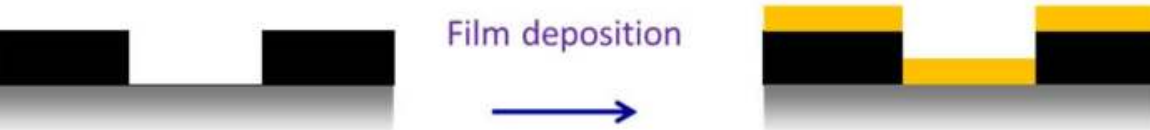

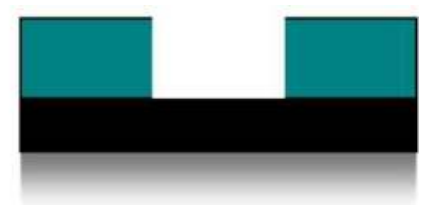
and patterning

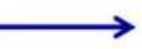

Peel-off 


\section{Lithographic fabrication using parylene-C}
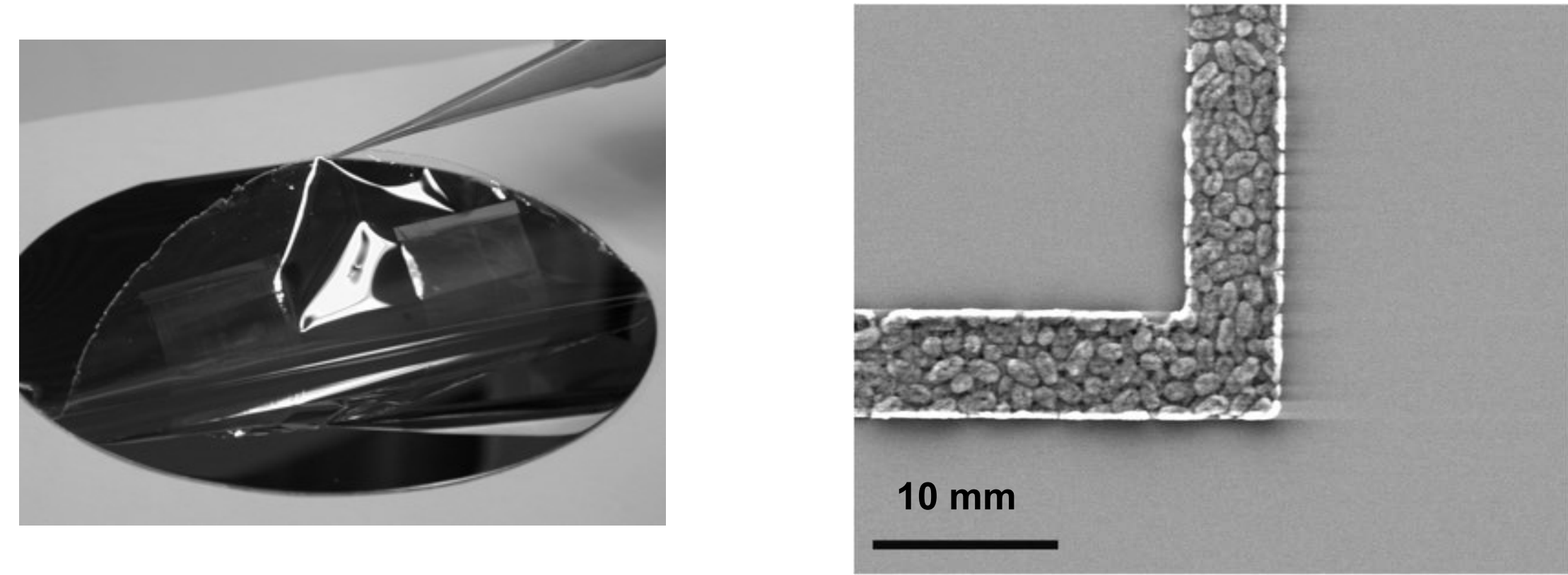

E. Coli 


\section{Microfabricated OECTs}
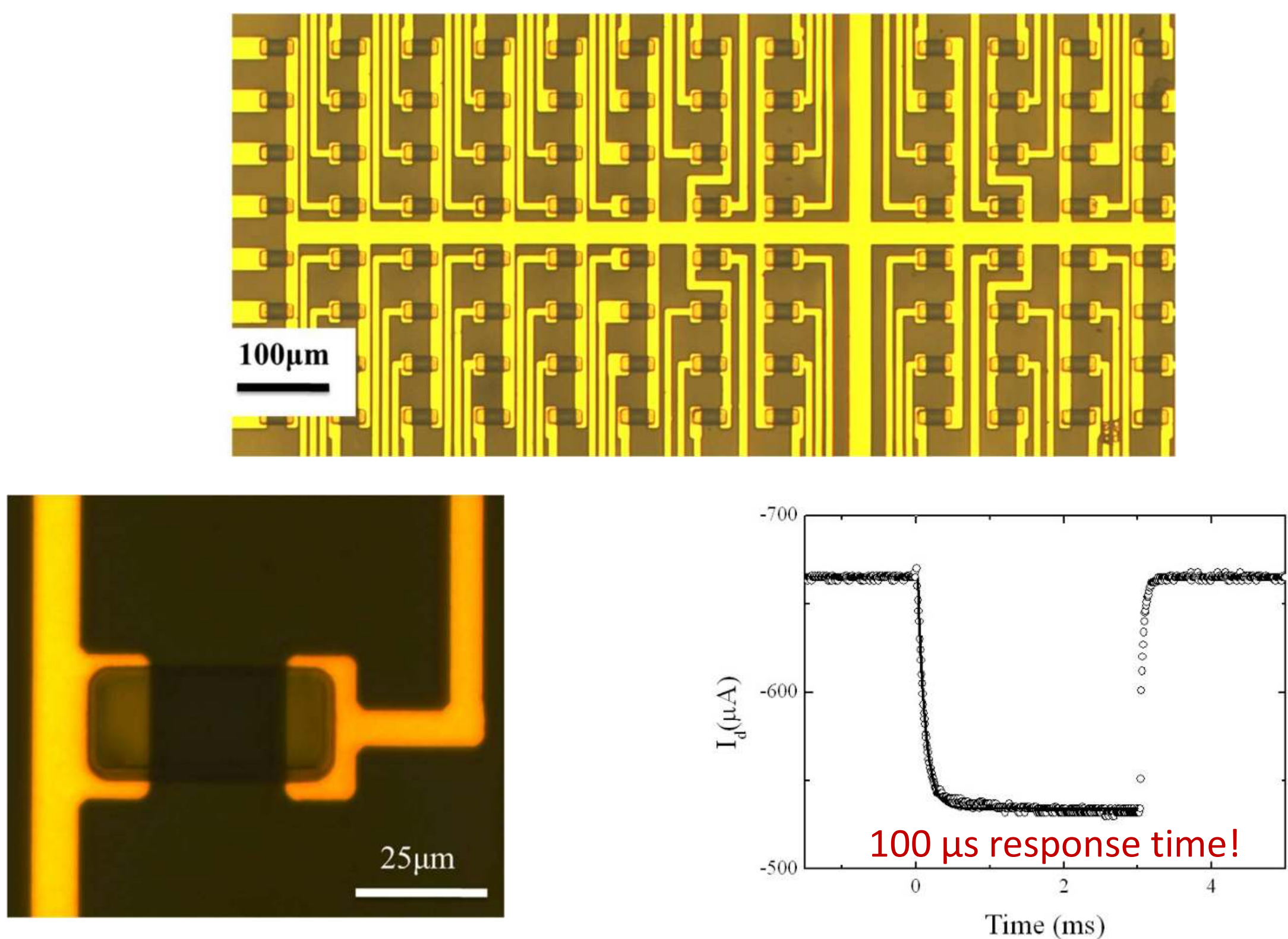

D. Khodagholy, M. Gurfinkel, E. Stavrinidou, P. Leleux, T. Herve, S. Sanaur, and G. G. Malliaras, Appl. Phys. Lett. 99 , 163304 (2011). 


\section{Integration with flexible substrates}
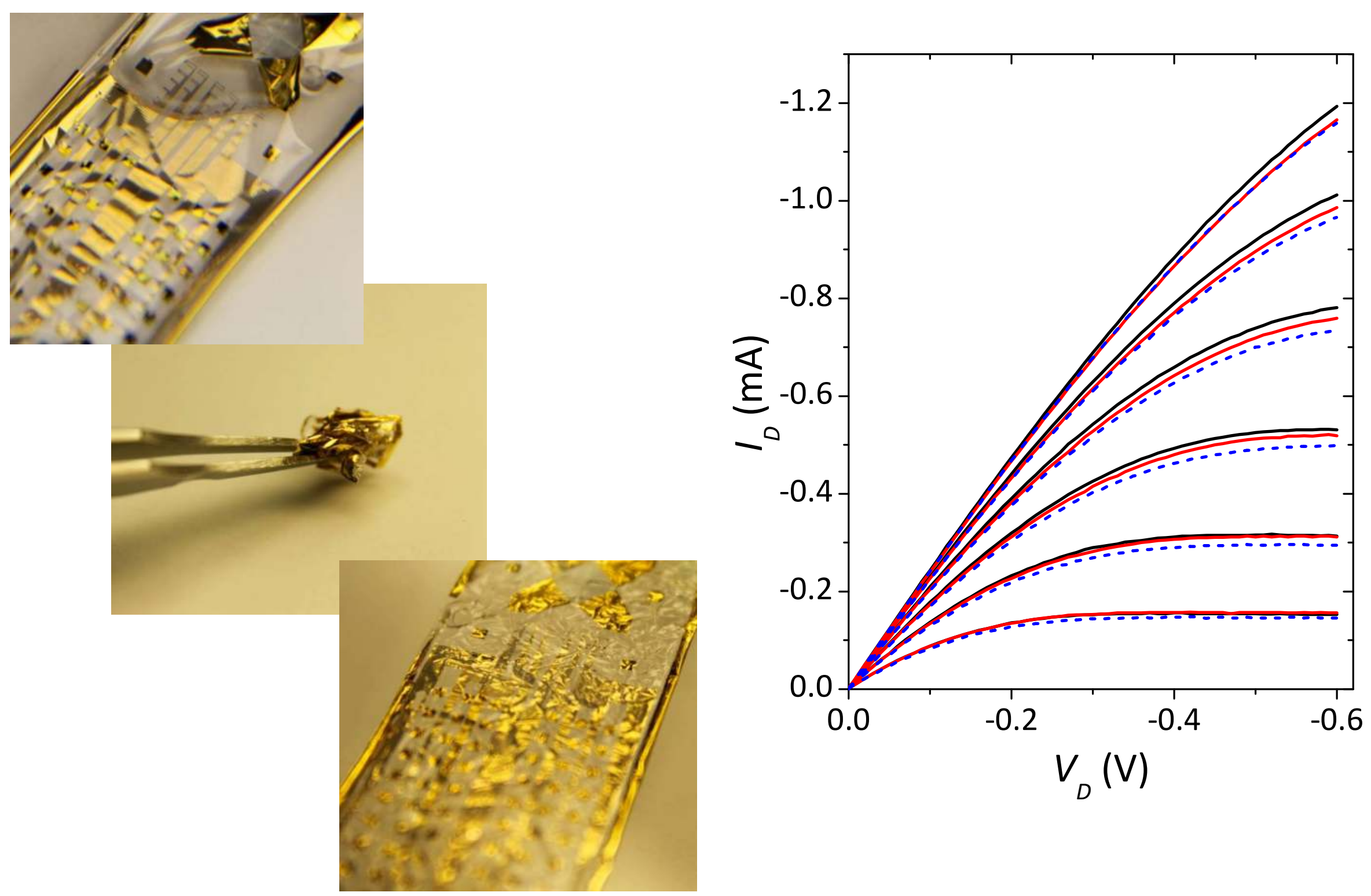


\section{Autoclave sterilisation}
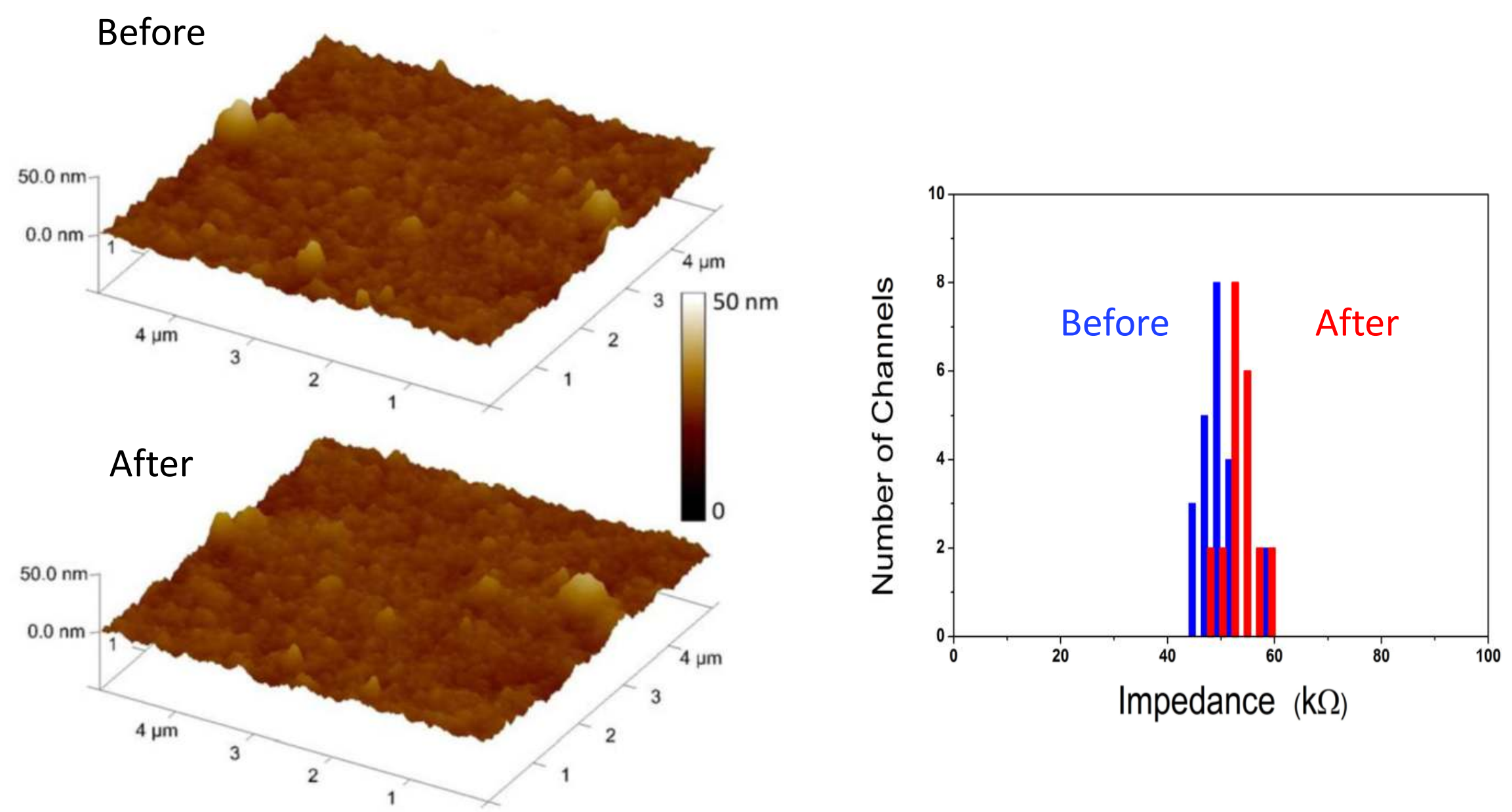

w/ Christophe Bernard (INSERM), Shadi Dayeh (UCSD) Adv. Healthcare Mater. 5, 3094 (2016). 


\section{Characteristics of OECTs}

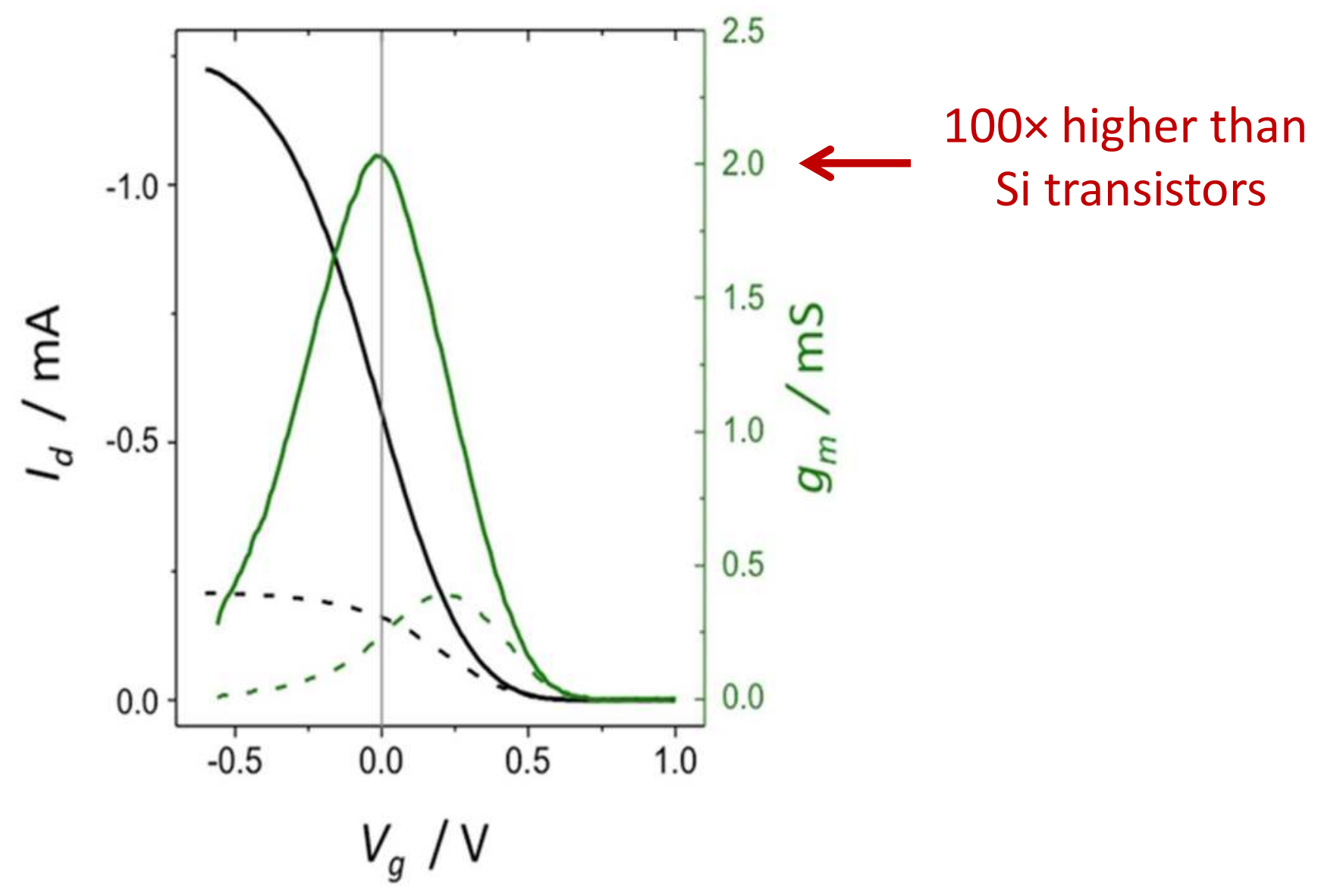




\section{In vivo recordings using transistors}
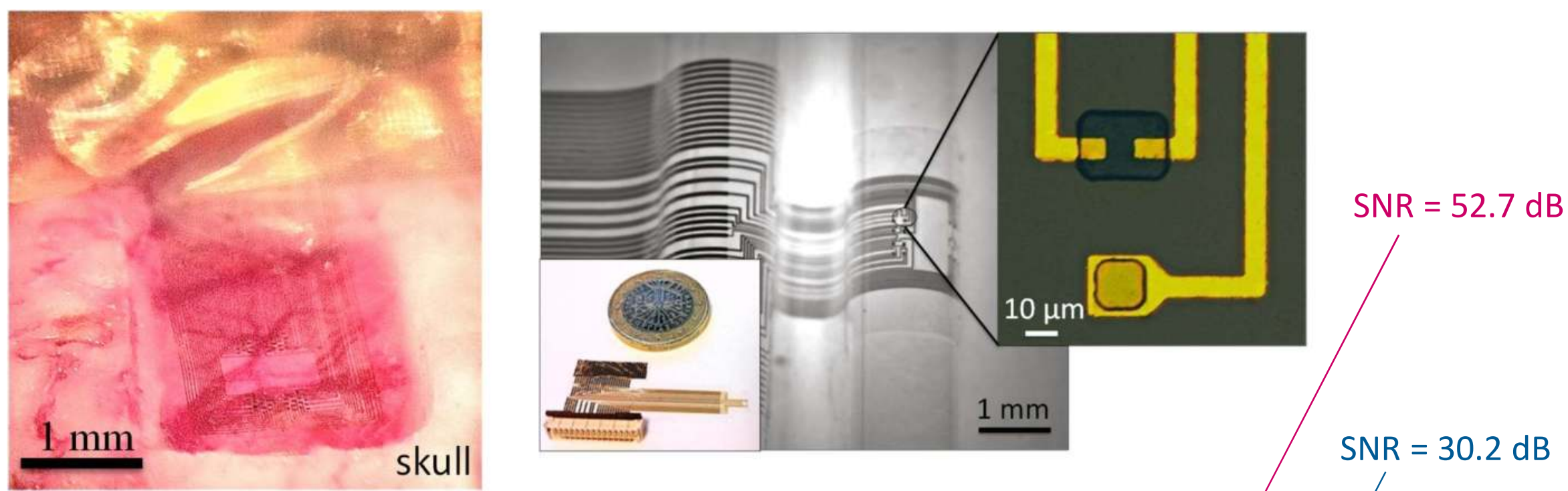

Transistor

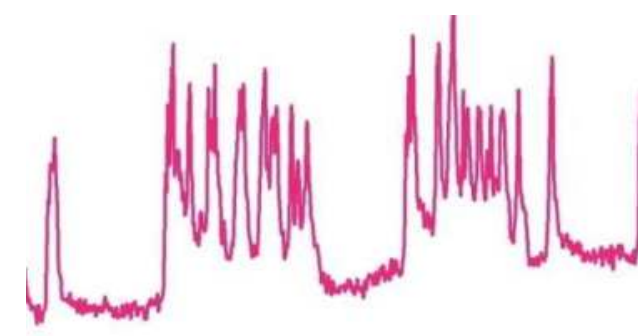

Electrode

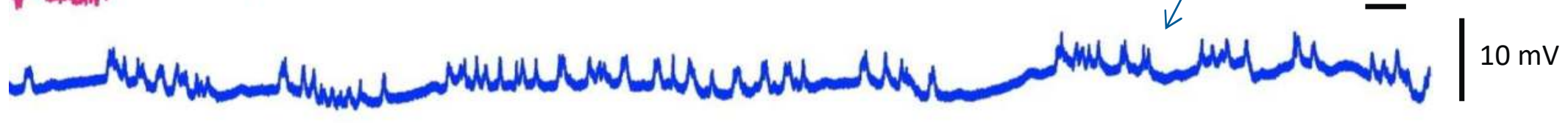

w/ Christophe Bernard (INSERM)

D. Khodagholy, T. Doublet, P. Quilichini, M. Gurfinkel, P. Leleux, A. Ghestem, E. Ismailova, T. Herve, S. Sanaur, C. Bernard, and G.G. Malliaras, Nature Comm. 4, 1575 (2013). 


\section{Transistors enable less invasive recordings}

Transistor
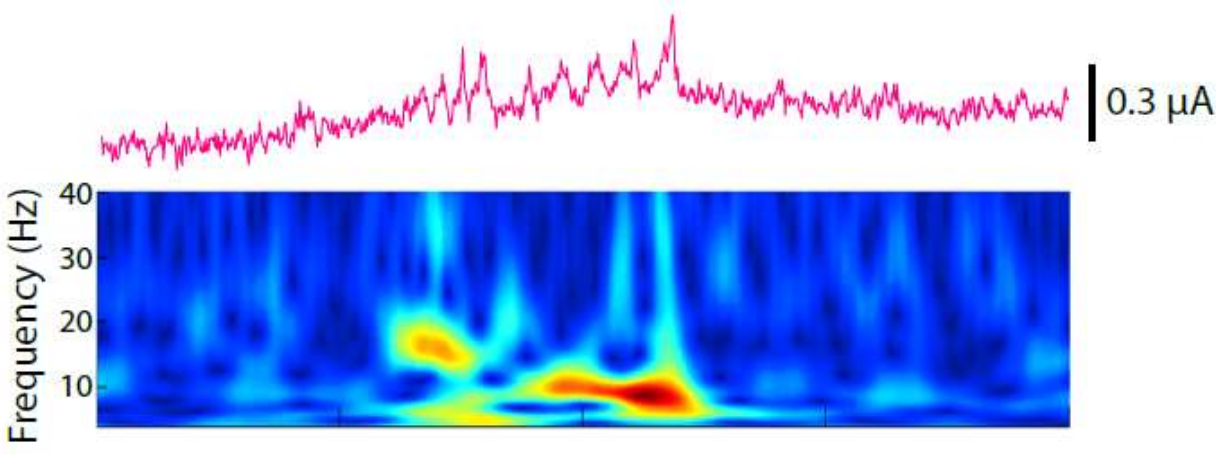

Surface electrode

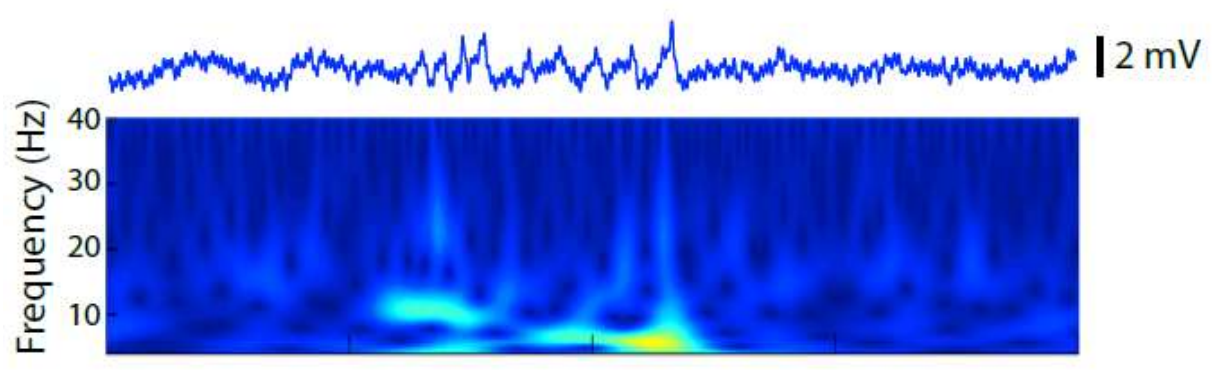

\section{Depth electrode}

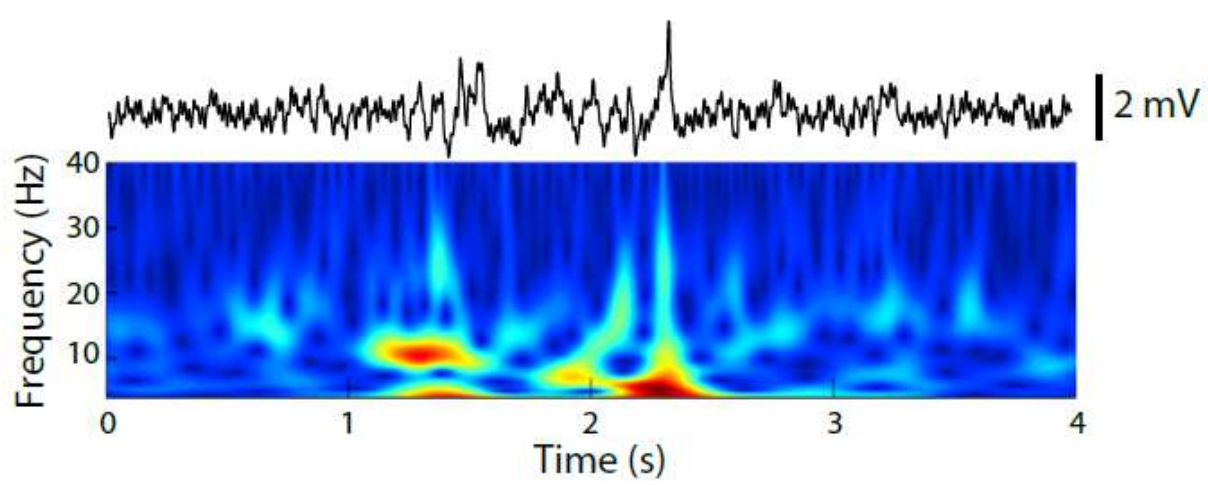

w/ Christophe Bernard (INSERM)

D. Khodagholy, T. Doublet, P. Quilichini, M. Gurfinkel, P. Leleux, A. Ghestem, E. Ismailova, T. Herve, S. Sanaur, C. Bernard, and G.G. Malliaras, Nature Comm. 4, 1575 (2013). 


\section{Multiplexed arrays for massive recordings}
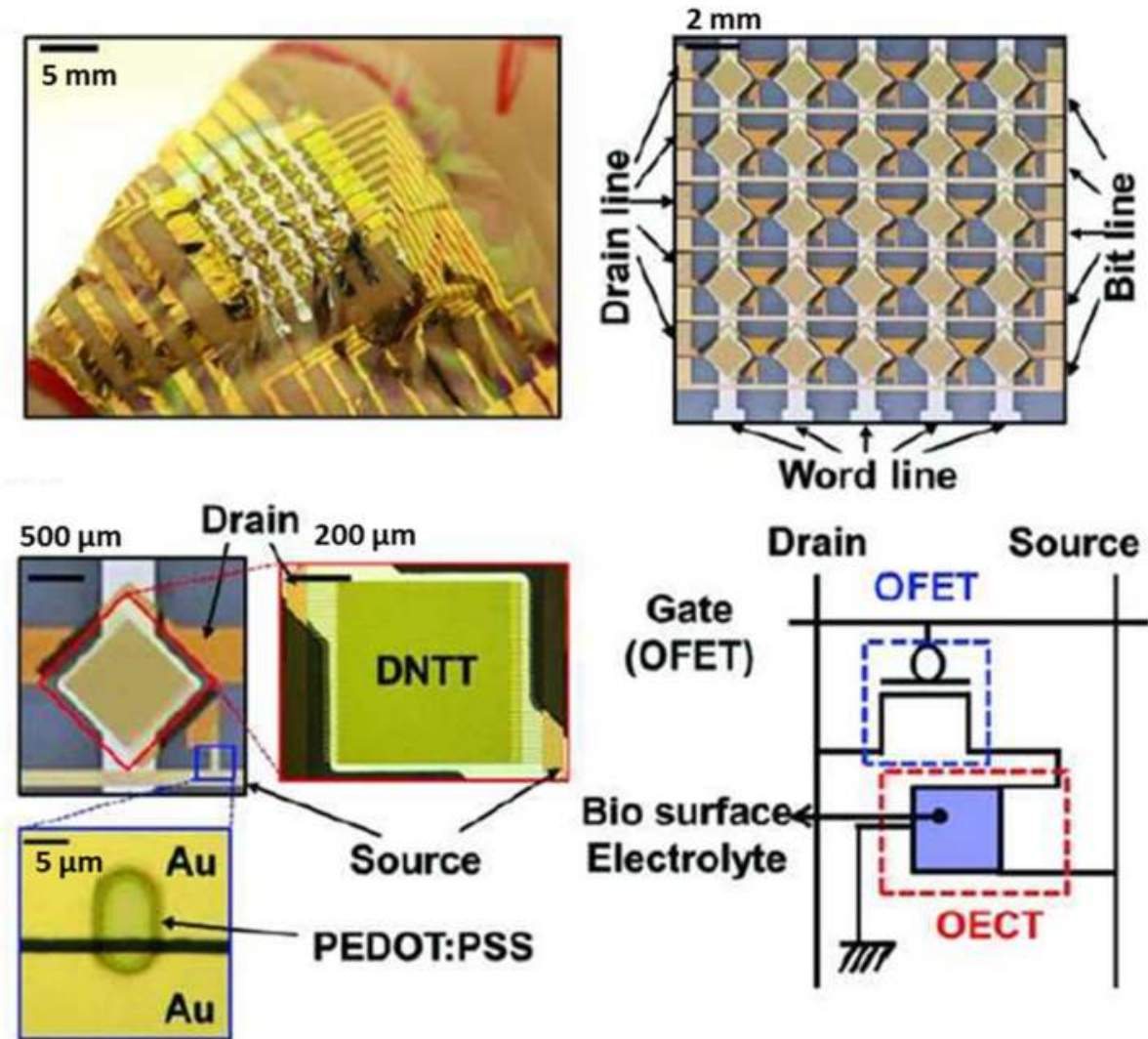

\section{w/ Takao Someya (Tokyo)}

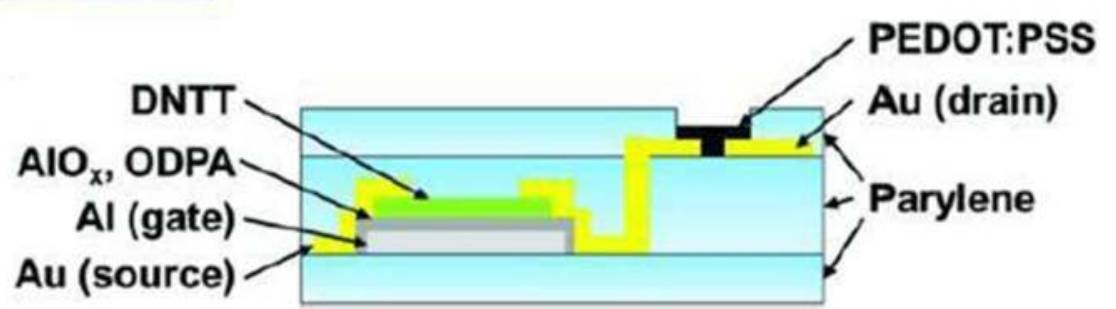

- W. Lee, D. Kim, J. Rivnay, N. Matsuhisa, T. Lonjaret, T. Yokota, H. Yawo, M. Sekino, G. Malliaras, T. Someya, Adv. Mater. 28, 9722 (2016).

- W. Lee, D. Kim, N. Matsuhisa, M. Nagase, M. Sekino, G.G. Malliaras, T. Yokota, and T. Someya, Proc. Natl. Acad. Sci. 114, 10554 (2017).

- W. Lee, S. Kobayashi, M. Nagase, Y. Jimbo, I. Saito, Y. Inoue, T. Yambe, M. Sekino, G.G. Malliaras, T. Yokota, M. Tanaka and T. Someya, Sci. Adv. 4 , eaau2426 (2018). 


\section{Insertion in the brain using removable shuttles}
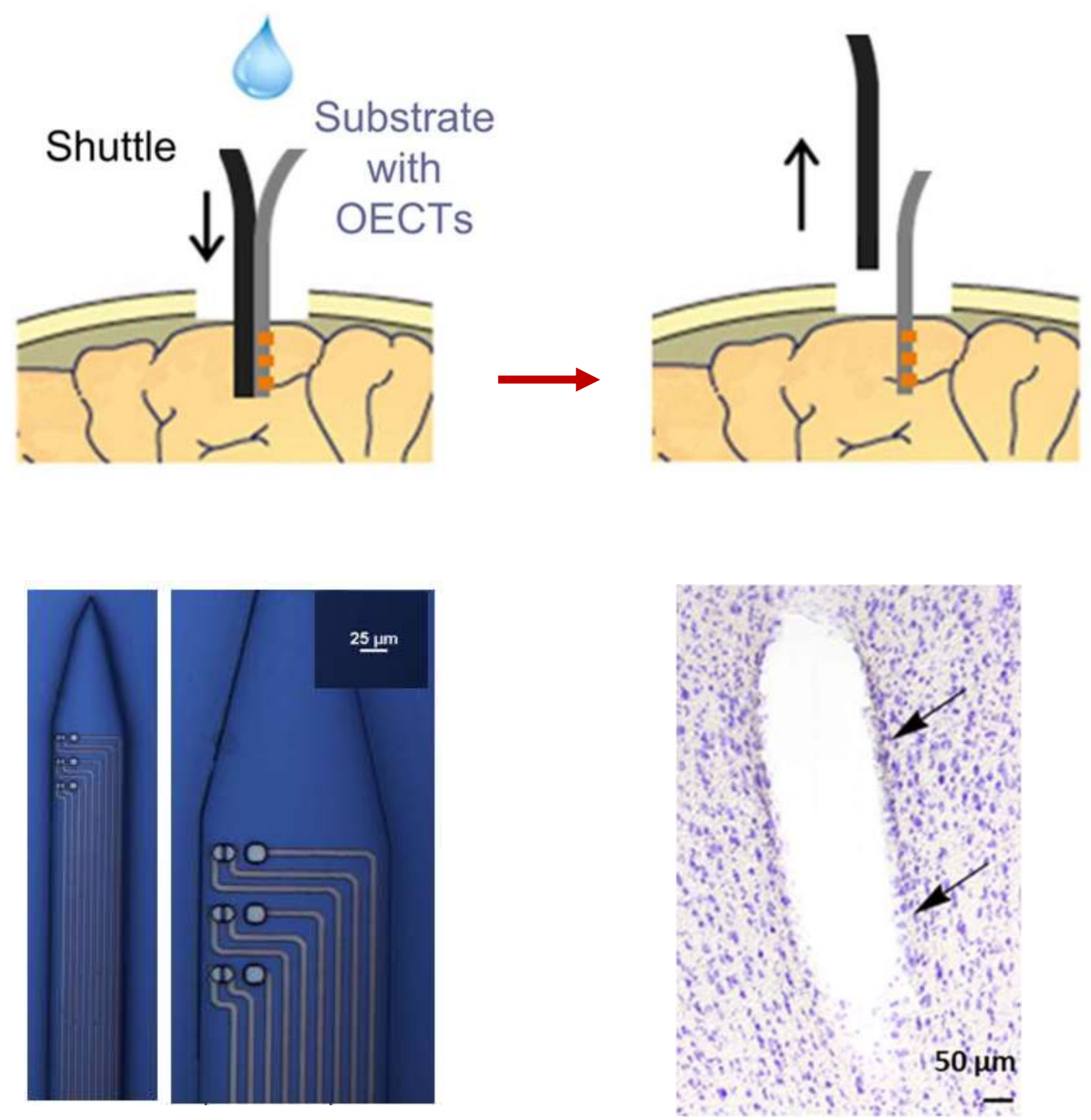

w/ Christophe Bernard (INSERM) 


\section{Model}

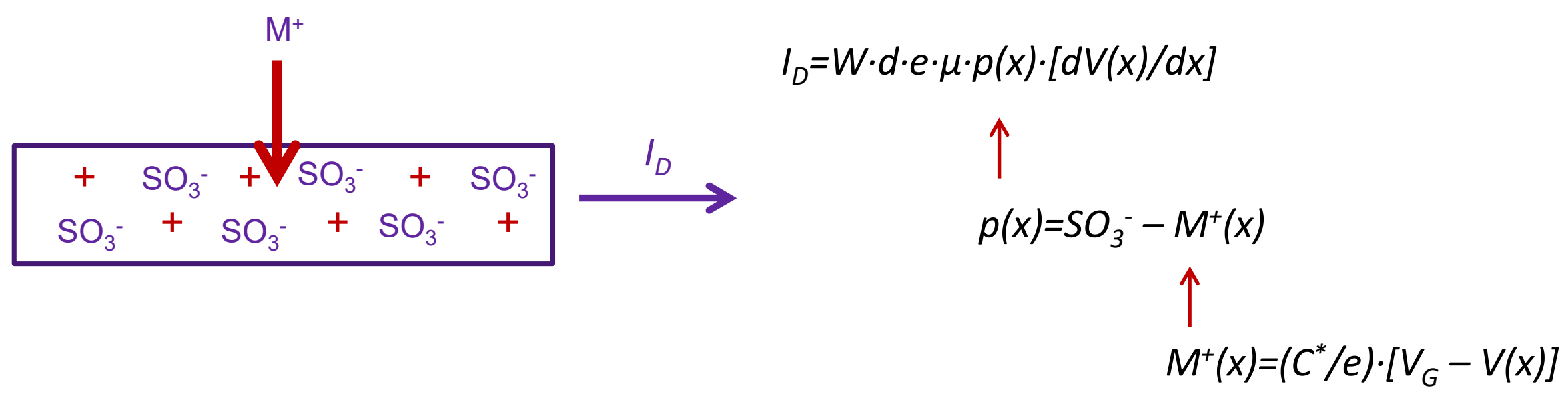

Integrating $I_{d}$ over the length of the channel:

$$
\begin{gathered}
I_{D}=(W \cdot d / L) \cdot \mu \cdot C^{*} \cdot\left[V_{T}-V_{G}+V_{D} / 2\right] \cdot V_{D} \\
V_{T}=e \cdot S O_{3}{ }^{-} / C^{*}
\end{gathered}
$$




\section{Scaling with geometry}
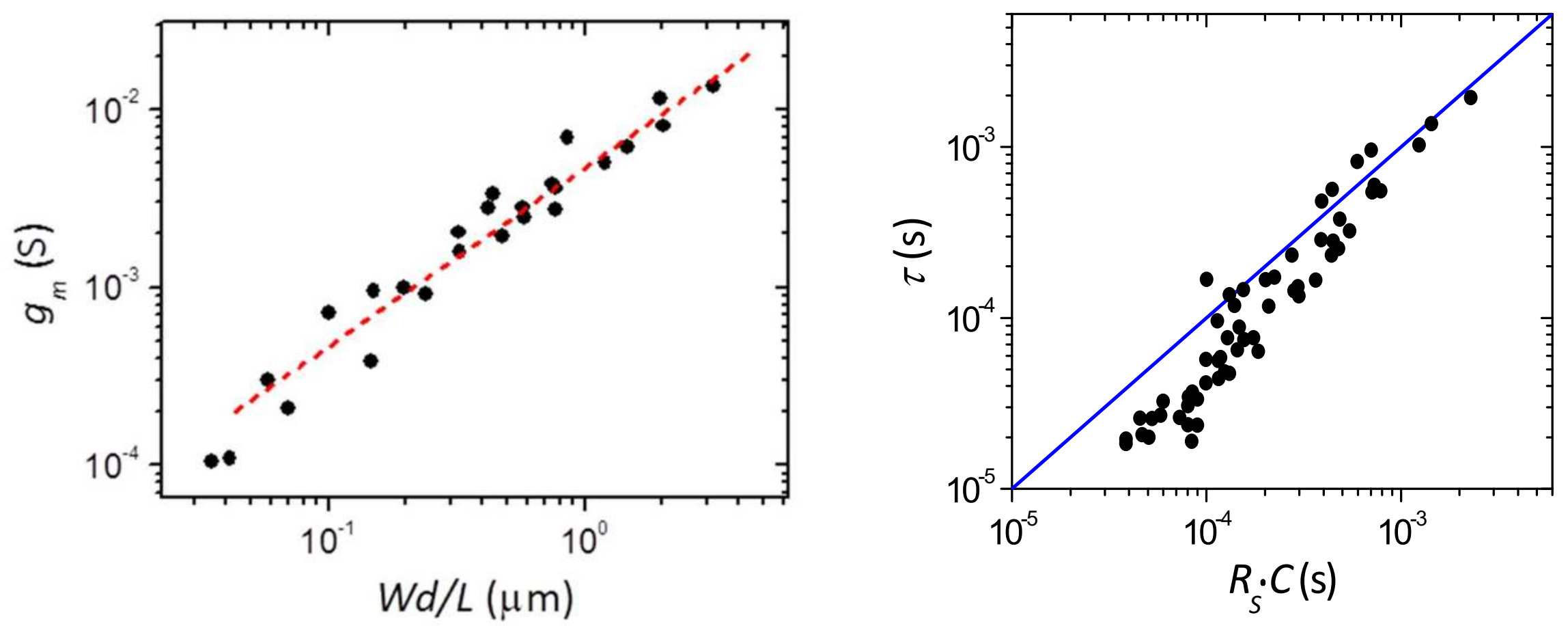

$$
g_{m}{ }^{S A T}=\left(W \cdot d /{ }_{L}\right) \cdot \mu \cdot C^{*} \cdot\left(V_{T}-V_{G}\right)
$$




\section{High transconductance means high SNR}

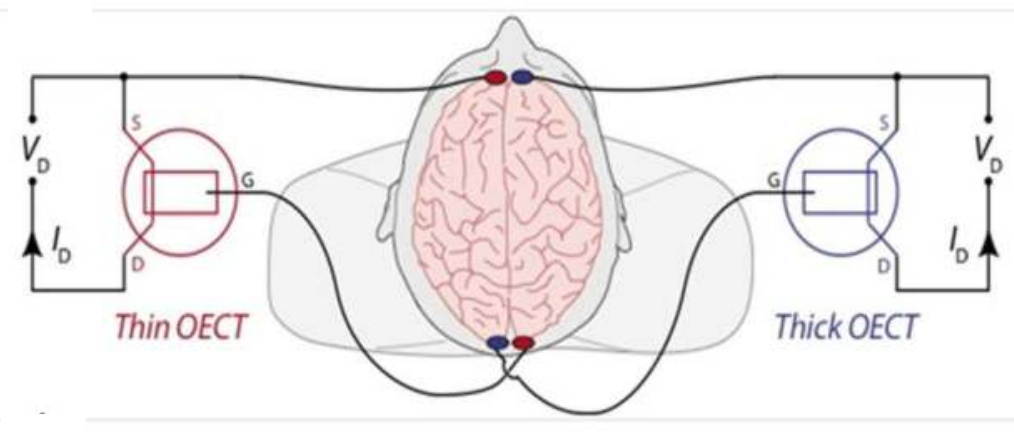

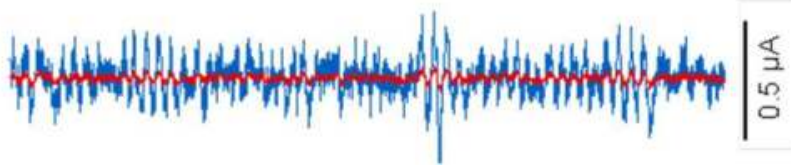

J. Rivnay, P. Leleux, M. Ferro, M. Sessolo, A. Williamson, D.A. Koutsouras, D. Khodagholy, M. Ramuz, X. Strakosas, R.M. Owens, C. Benar, J.-M. Badier C. Bernard, and G.G. Malliaras, SCIENCE Advances 1, e1400251 (2015).

w/ Christian Benar, Jean-Michel Badier (INSERM)

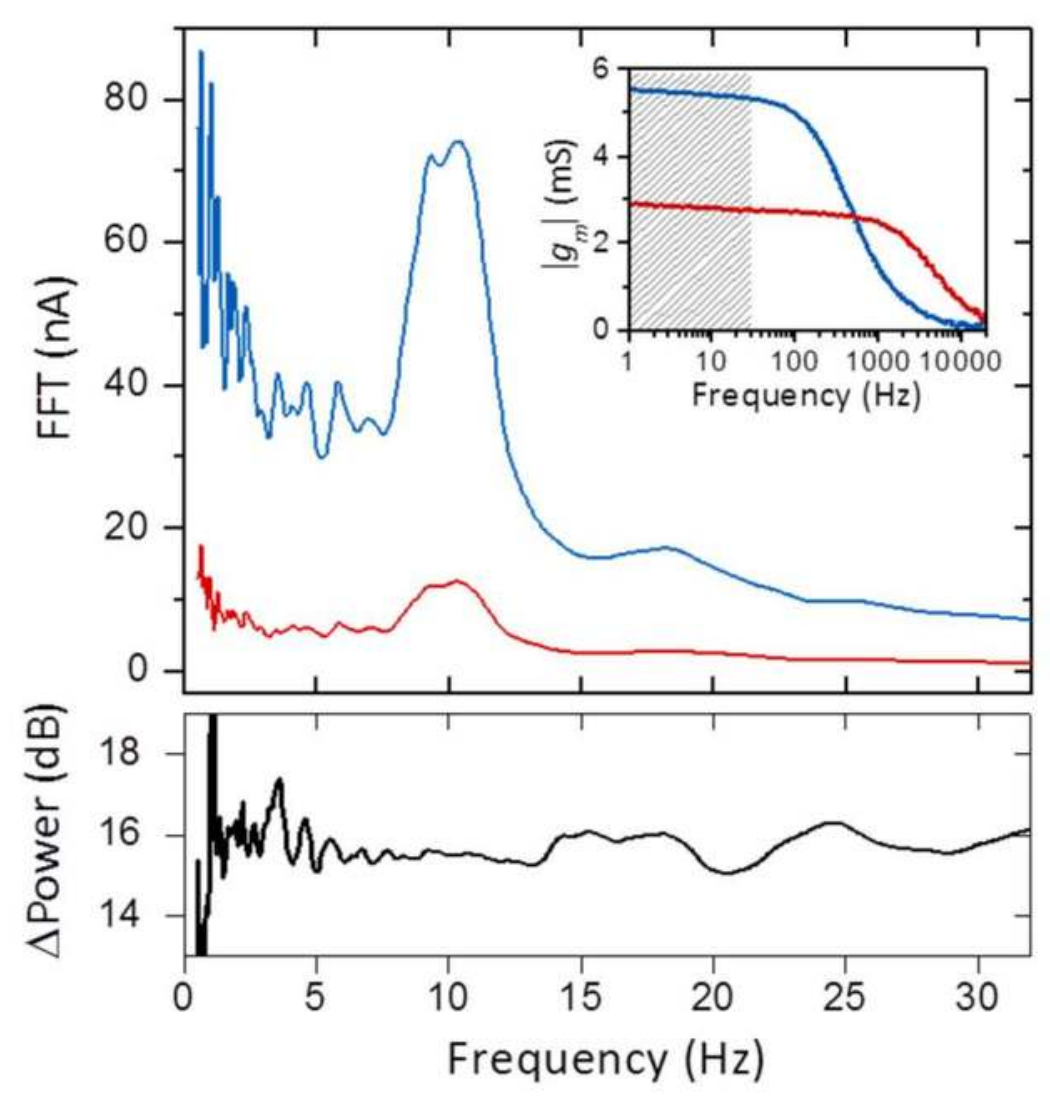




\section{Optimizing by means of materials processing}
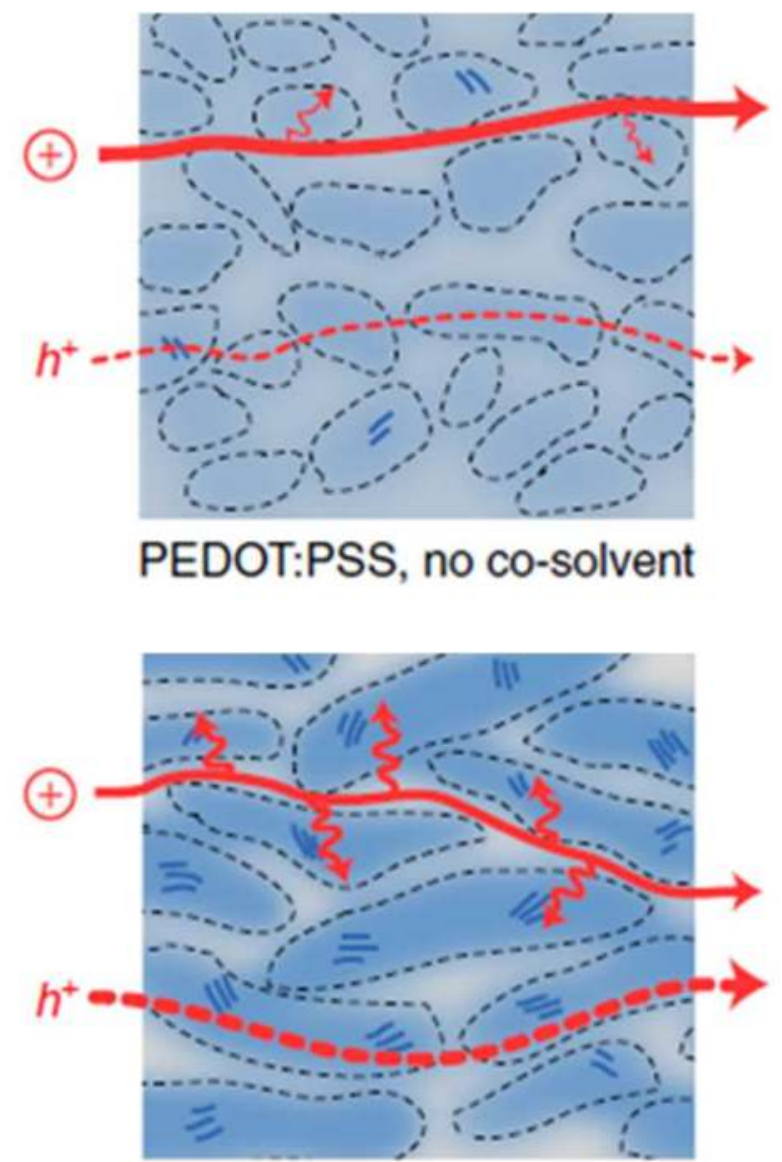

PEDOT:PSS + ethylene glycol

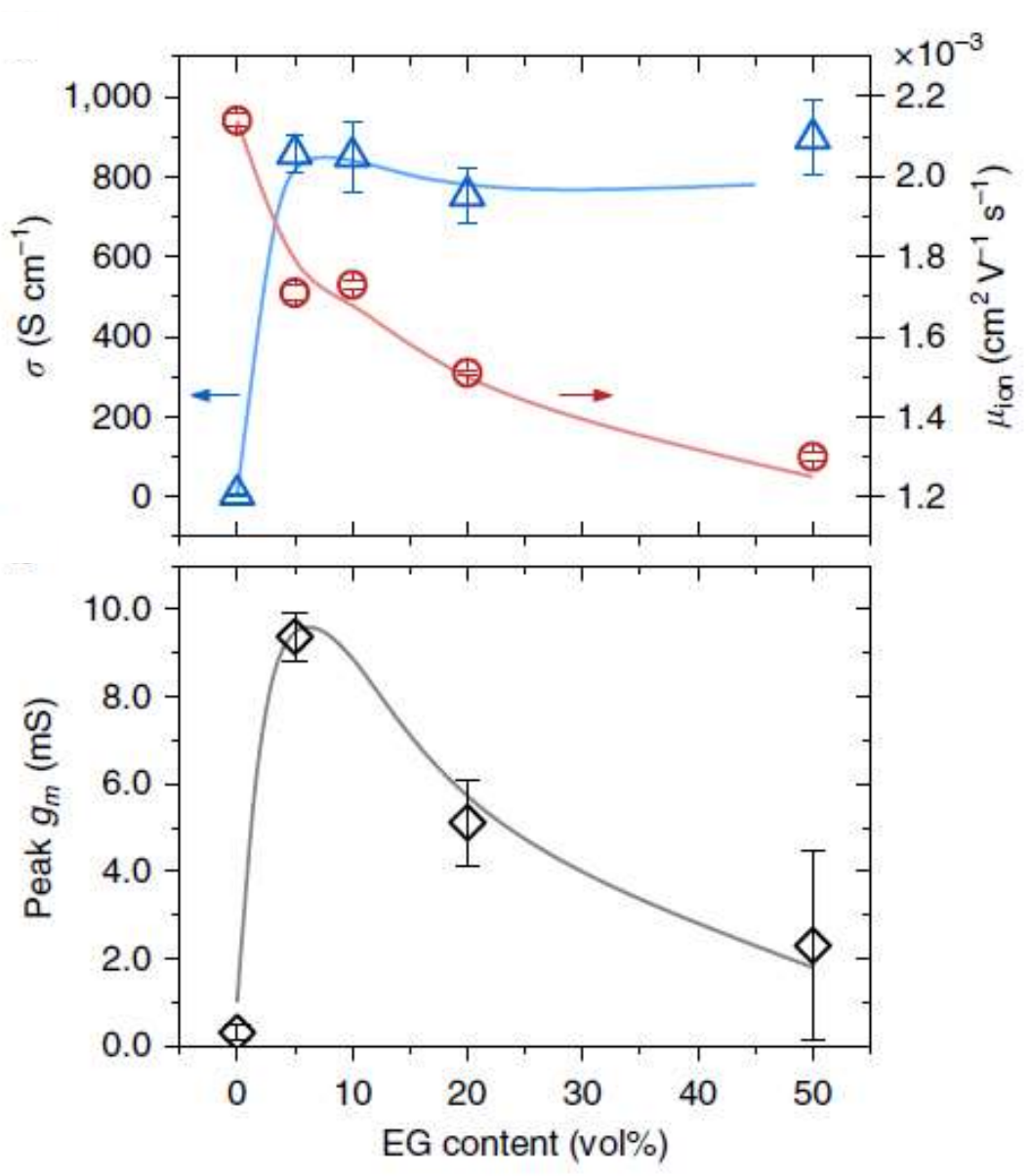




\section{$\mu \cdot C^{*}$ of different materials}

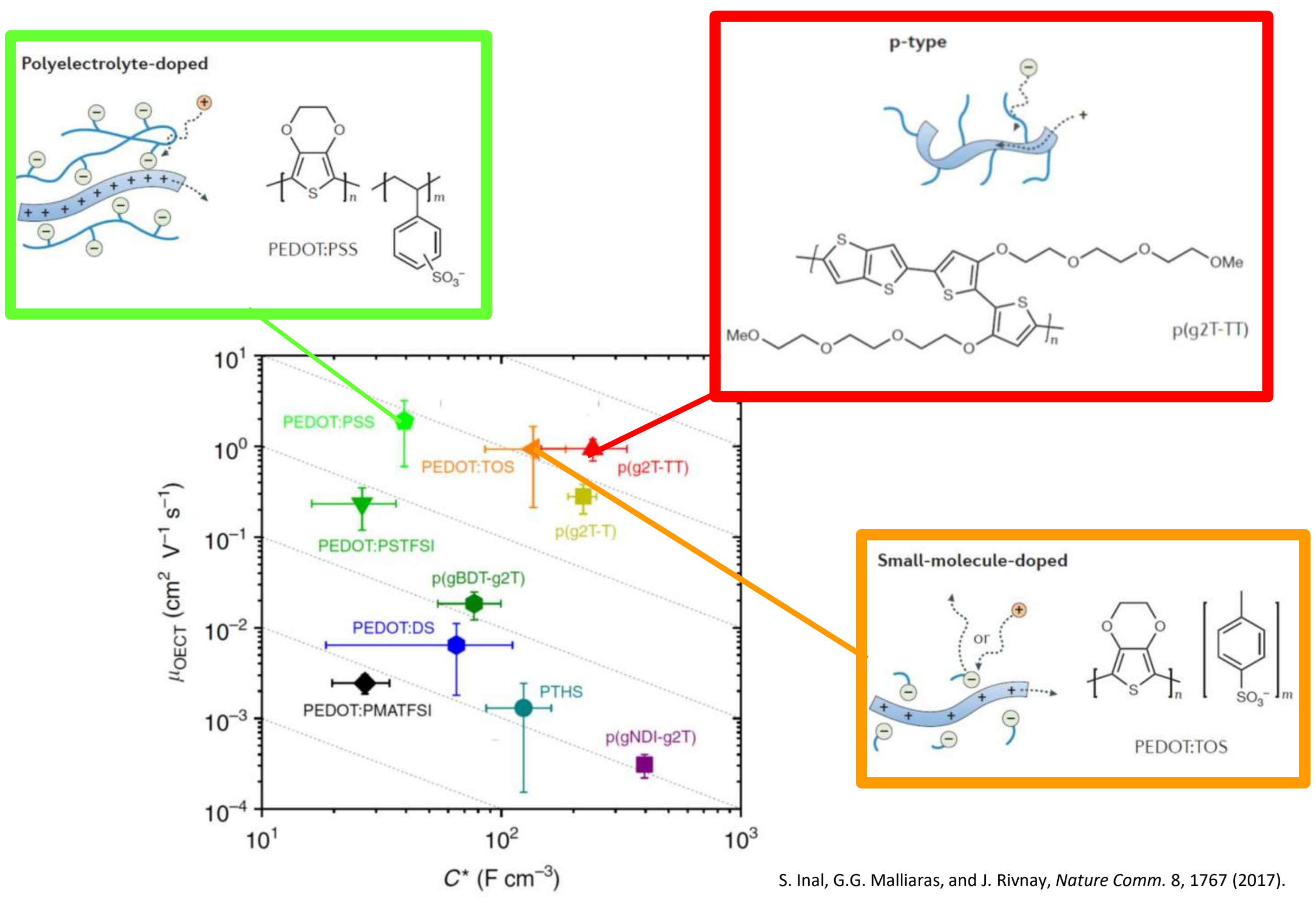




\section{Conclusions}

- Organic electrochemical transistors leverage volumetric ions transport to yield high transconductance.

- They are amplifying transducers that yield better signal-to-noise ratio recordings than electrodes. They allow to look deeper in the brain.

- PEDOT:PSS is a champion material as it offers high hole mobility coupled with facile ion injection and transport. Better materials require:

- High electronic carrier mobility

- High volumetric capacitance

A winning strategy: efficient $\pi$-conjugated backbones with hydrophilic side chains 


\section{Bioelectronics Acknowledgements}

Co-leader: Damiano Barone Vincenzo Curto Chris Proctor Alexandra Rutz Alej Carnicer-Lombarte Sanggil Han Chen Jiang Johannes Jurke Anastasios Polyravas Tanya Mangoma Amy Rochford Shao-Tuan Chen Ben Woodington Sagnik Middya

Tobias Naegele Elise Jenkins Santiago Velasco-Bosom Yi-Lin Yu De-Shaine Murray Will Halfpenny Louise Aumont Roberto Pezone Shunsuke Yamamoto Menglun Zhang lasonas Triantis Eleftheria Batagianni Theana Johnson

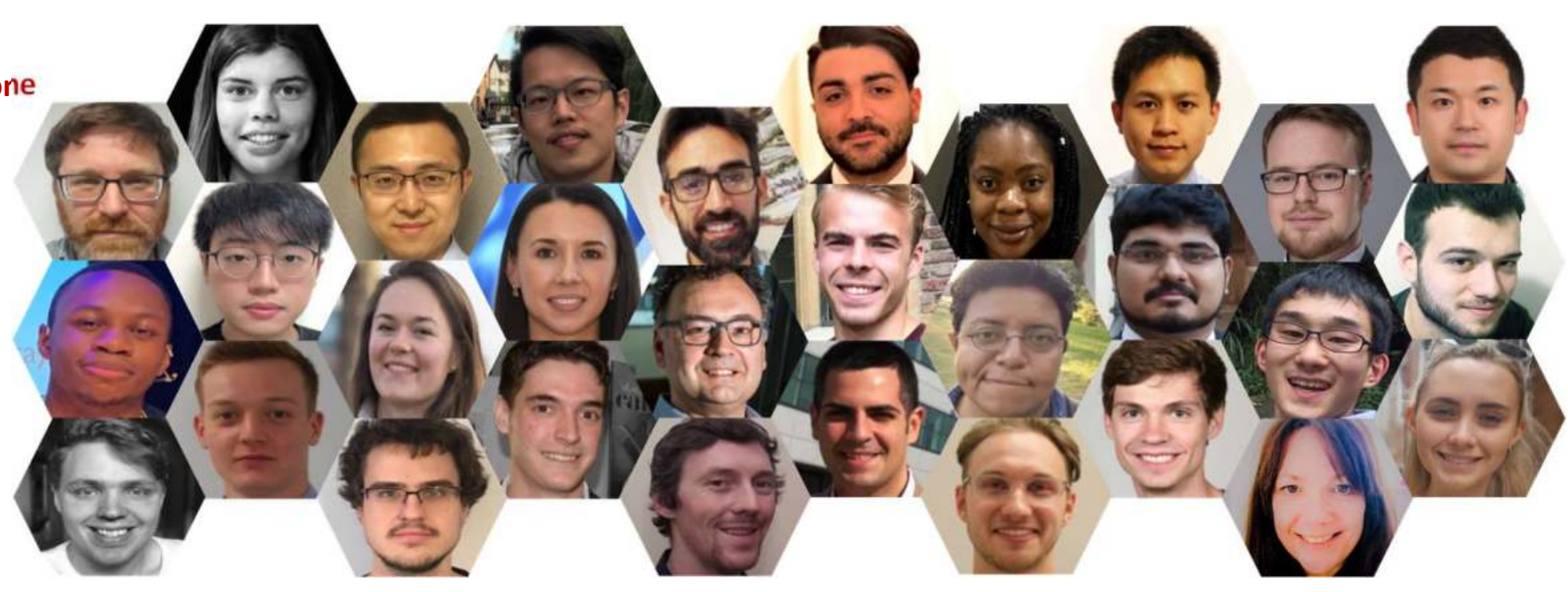

\section{University of Cambridge}

Roisin Owens (Chemical Engineering)

Gabi Kaminski (Chemical Engineering) David Fairen-Jimenez (Chemical Engineering) Ronan Daly (Institute for Manufacturing) Oren Scherman (Melville Laboratory) Clare Grey (Chemistry) Jenny Morton (PDN)

Manohar Bance (Clinical Neurosciences) Stephen Price (Clinical Neurosciences) Michael Lee (Medicine) Richard Gilbertson (Oncology)

\section{Other Collaborators}

Mary Donahue, Rodney O'Connor (Mines St Etienne) Adam Williamson, Christophe Bernard (Aix Marseille) Sahika Inal, lain McCulloch (KAUST)

Daniel Simon, Magnus Berggren (Linköping University) Jonathan Rivnay (Northwestern University) Dion Khodagholy (Columbia University) Takao Someya (University of Tokyo) David Martin (University of Delaware) Robert McLeod (University of Colorado) Martyn Boutelle (Imperial College London) David Mecerreyes (University of Basque Country)
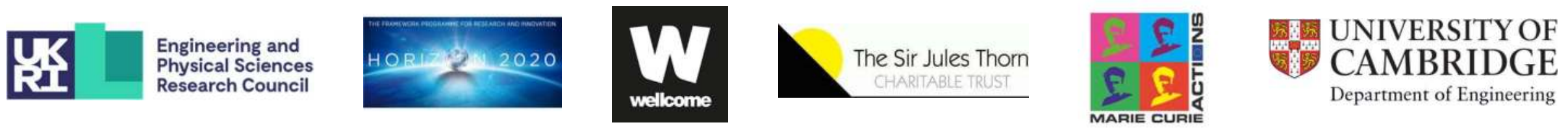